Pacific Journal of Mathematic 


\title{
FUNDAMENTAL GROUPS OF COMPACT COMPLETE LOCALLY AFFINE COMPLEX SURFACES, II
}

\author{
JOHN SCHEUNEMAN
}

The present article is a continuation of a recent paper by J. P. Fillmore and the author on properly-acting groups $\Gamma$ of complex affine motions of $C^{2}$ such that $\Gamma \backslash C^{2}$ is compact. In that paper, it was proved that such a group has a normal subgroup $\Gamma_{0}$ of finite index which is either free abelian of rank four or has generators $A, B, C, D$, with relations

$$
A B A^{-1} B^{-1}=C^{k}(k \geqq 1)
$$

and $C$ and $D$ central.

Here we build on this description up to finite index to determine the groups $\Gamma$ themselves.

1. Introduction. A complete locally affine complex surface $X$ has an orbit-space representation $X=\Gamma \backslash C^{2}$, where the fundamental group $\Gamma$ of $X$ is a properly-acting group of complex affine transformations of $C^{2}$. Two such surfaces $\Gamma \backslash C^{2}$ and $\Gamma^{\prime} \backslash C^{2}$ are isomorphic if and only if $\Gamma$ and $\Gamma^{\prime}$ are conjugate subgroups of the group $A(2$, $C)$ of all complex affine motions of $C^{2}$. Elements of $A(2, C)$ are taken as nonsingular complex matrices $\left(\begin{array}{lll}a & b & r \\ c & d & s \\ 0 & 0 & 1\end{array}\right)$ and elements of $C^{2}$ are taken as column vectors $\left(\begin{array}{l}x \\ y \\ 1\end{array}\right)$; then $A(2, C)$ acts on the left on $C^{2}$ in the usual way.

Fillmore and Scheuneman [2] have shown the following:

THEOREM 1.1. Let $\Gamma \backslash C^{2}$ be a compact complete locally affine complex surface. Then:

( i ) $\Gamma$ is conjugate in $A(2, C)$ to a subgroup of the group $G$ of all matrices of the form $\left(\begin{array}{lll}1 & b & r \\ 0 & d & s \\ 0 & 0 & 1\end{array}\right)$, and hence may be considered $a$ subgroup of $G$;

(ii) The homomorphism $\left(\begin{array}{lll}1 & b & r \\ 0 & d & s \\ 0 & 0 & 1\end{array}\right) \mapsto d$, when restricted to $\Gamma$, has a kernel $\Gamma_{0}$ which is either free abelian of rank four, or has generators $A, B, C, D$ with relations $A B A^{-1} B^{-1}=C^{k}(k \geqq 1)$ and $C$ and $D$ central in $\Gamma_{0}$;

(iii) The image of $\Gamma$ under the above homomorphism is a finite cyclic group of order $t=1,2,3,4,5,6,8,10$, or 12 . 
In view of this result, we immediately obtain a description as follows: $\Gamma$ is generated by $S, A, B, C, D$, where $S$ is a preimage of a generator of $\Gamma / \Gamma_{0}, A, B, C, D$ is a set of generators of $\Gamma_{0}, S^{t} \in \Gamma_{0}$, and $S \Gamma_{0} S^{-1}=\Gamma_{0}$. This description is too general, for not all groups satisfying these conditions are of the kind we are interested in here. It must be refined by imposing the conditions that $\Gamma$ be a subgroup of the group $G$ of 1.1 (i), and that it act properly on $C^{2}$ and have compact orbit space.

In $\S 2$, we normalize $\Gamma_{0}$ up to conjugation. This normalization leads to the elimination of the cases $t=5,8,10,12$ in 1.1 (iii). In succeeding sections we describe the generators and relations for $\Gamma$ using the normalization. Our study of the problem at hand uses a blend of abstract group theory, which is more efficient than matrix calculations, together with calculations involving embedded groups (groups of matrices), this being an essential ingredient in the problem.

2. Normalization of $\Gamma_{0}$.

LEMmA 2.1. Let $T=\left(\begin{array}{lll}1 & b & r \\ 0 & d & s \\ 0 & 0 & 1\end{array}\right)$ in $A(2, C)$ be different from the identity. Then $T$ has a fixed point in $C^{2}$ if and only if

(1) $b \neq 0$ and $s=0$, in case $d=1$;

(2) $b s-(d-1) r=0$, in case $d \neq 1$.

Proof. A point $(x, y)$ of $C^{2}$ is fixed under $T$ if $x+b y+r=x$ and $d y+s=y$. It is easy to check that if such a point exists, the conditions hold. Conversely, if the conditions hold, the points $(0,-r / b)$ and $(0,-s /(d-1))$ are fixed in the respective cases.

Let $\Gamma_{0}$ denote a subgroup of

$$
G_{0}=\left\{\left(\begin{array}{lll}
1 & b & r \\
0 & 1 & s \\
0 & 0 & 1
\end{array}\right) \mid b, r, s \text { complex }\right\} \subset A(2, C)
$$

which acts properly on $C^{2}$ and such that $\Gamma_{0} \mid C^{2}$ is compact.

Proposition 2.2.

(i) Suppose $\Gamma_{0}$ is generated by four element $A, B, C$, and $D$ with relations $A B A^{-1} B^{-1}=C^{k}$ and $C$ and $D$ central for some fixed $k \geqq 1$. Then $\Gamma_{0}$ is conjugate in $A(2, C)$ to a group where

$$
A=\left(\begin{array}{lll}
1 & \lambda & 0 \\
0 & 1 & 1 \\
0 & 0 & 1
\end{array}\right), B=\left(\begin{array}{ccc}
1 & \lambda b-k & 0 \\
0 & 1 & b \\
0 & 0 & 1
\end{array}\right), C=\left(\begin{array}{lll}
1 & 0 & 1 \\
0 & 1 & 0 \\
0 & 0 & 1
\end{array}\right) \text {, and } D=\left(\begin{array}{lll}
1 & 0 & d^{\prime} \\
0 & 1 & 0 \\
0 & 0 & 1
\end{array}\right) \text {. }
$$


(ii) Suppose $\Gamma_{0}$ is free abelian of rank four with generators $A, B, C$, and $D$. Then $\Gamma_{0}$ is conjugate in $A(2, C)$ to a group where

$$
A=\left(\begin{array}{lll}
1 & \lambda & a^{\prime} \\
0 & 1 & 1 \\
0 & 0 & 1
\end{array}\right), B=\left(\begin{array}{ccc}
1 & \lambda b & b^{\prime} \\
0 & 1 & b \\
0 & 0 & 1
\end{array}\right), C=\left(\begin{array}{ccc}
1 & \lambda c & c^{\prime} \\
0 & 1 & c \\
0 & 0 & 1
\end{array}\right) \text {, and } D=\left(\begin{array}{ccc}
1 & \lambda d & d^{\prime} \\
0 & 1 & d \\
0 & 0 & 1
\end{array}\right) \text {. }
$$

Proof. (i) The commutator subgroup of $G$ consists of matrices of the form $\left(\begin{array}{lll}1 & 0 & * \\ 0 & 1 & 0 \\ 0 & 0 & 1\end{array}\right)$, thus $C^{k}$ is a matrix of this form. The 12- and 23- entries of matrices in $G$ add when the matrices are multiplied, so we have that $C$ itself is of the form $C=\left(\begin{array}{lll}1 & 0 & c^{\prime} \\ 0 & 1 & 0 \\ 0 & 0 & 1\end{array}\right)$ with $c^{\prime} \neq 0$. Conjugate $C$ by $P=\left(\begin{array}{ccc}1 / c^{\prime} & 0 & 0 \\ 0 & 1 & 0 \\ 0 & 0 & 1\end{array}\right)$ in $A(2, C)$; this gives $P C P^{-1}=\left(\begin{array}{lll}1 & 0 & 1 \\ 0 & 1 & 0 \\ 0 & 0 & 1\end{array}\right)$ and preserves $G, P G P^{-1}=G$. Hence we may assume that $C$ has $c^{\prime}=$ 1. Note that $C$ is central. Set $A=\left(\begin{array}{lll}1 & \lambda & a^{\prime} \\ 0 & 1 & a \\ 0 & 0 & 1\end{array}\right), B=\left(\begin{array}{lll}1 & \mu & b^{\prime} \\ 0 & 1 & b \\ 0 & 0 & 1\end{array}\right)$, and $D=$ $\left(\begin{array}{lll}1 & \delta & d^{\prime} \\ 0 & 1 & d \\ 0 & 0 & 1\end{array}\right)$. From $A B A^{1} B^{-1}=C^{k}$ we obtain $\lambda b-\mu a=k \neq 0$. Since $D$ commutes with $A$ and $B$, we have, respectively, $\lambda d=\delta a$ and $\mu d=$ $\delta b$. Where $d \neq 0$, we would have $k=\lambda b-\mu a=(\delta a b-\delta b a) / d=0$, a contradiction. Hence, $d=0$. By 2.1 we must have $\delta=0$ also. Now $C$ and $D$ have the desired form. From Lemma 3.1 of [2] we have $a \neq 0$. Conjugate by $P=\left(\begin{array}{ccc}1 & -a^{\prime} / a & 0 \\ 0 & 1 / a & 0 \\ 0 & 0 & 1\end{array}\right) . \quad P$ commutes with $C$ and $D$, so these are unchanged; $P A P^{-1}=\left(\begin{array}{ccc}1 & \lambda a & 0 \\ 0 & 1 & 1 \\ 0 & 0 & 1\end{array}\right)$, and $P B P^{-1}$ has the same form as $B$. Hence we may assume $A$ has $a=1$ and $a^{\prime}=0$. Set $P=\left(\begin{array}{ccc}1 & \lambda s & r \\ 0 & 1 & s \\ 0 & 0 & 1\end{array}\right) . \quad P$ commutes with $A, C$, and $D$, while $P B P^{-1}=$ $\left(\begin{array}{ccc}1 & \mu & b^{\prime}+(\lambda b-\mu) s \\ 0 & 1 & b \\ 0 & 0 & 1\end{array}\right)$. Now $A B A^{-1} B^{-1}=C^{k}$ has its 13-entry equal to $\lambda b-\mu=k \neq 0$. Set $s=-b^{\prime} /(\lambda b-\mu)$ and $r=0$. Conjugation by $P$ leaves $A, C$, and $D$ unchanged and replaces $B$ by a matrix of the same form with $b^{\prime}=0$. We still have $\lambda b-\mu=k$, so $\mu=\lambda b-k$.

(ii) Select an arbitrary set of generators of $\Gamma_{0}$. By Lemma 3.1 of [2], one of these generators must have its 23-entry different from zero. Call this one $A=\left(\begin{array}{lll}1 & \lambda & a^{\prime} \\ 0 & 1 & a \\ 0 & 0 & 1\end{array}\right)$. For $P=\left(\begin{array}{ccc}1 & 0 & 0 \\ 0 & 1 / a & 0 \\ 0 & 0 & 1\end{array}\right)$ we have 
$P A P^{-1}=\left(\begin{array}{ccc}1 & \lambda a & a^{\prime} \\ 0 & 1 & 1 \\ 0 & 0 & 1\end{array}\right)$. Hence we may take $a=1$, so $A=\left(\begin{array}{ccc}1 & \lambda & a^{\prime} \\ 0 & 1 & 1 \\ 0 & 0 & 1\end{array}\right)$. A matrix of $G$ which commutes with $A$ necessarily has the form $\left(\begin{array}{ccc}1 & \lambda x & x^{\prime} \\ 0 & 1 & x \\ 0 & 0 & 1\end{array}\right)$, and all such matrices commute. Hence $B, C$, and $D$ have the form indicated.

It is easily verified that conversely, if $A, B, C, D$ are as in 2.2, then the group they generate acts properly on $C^{2}$ provided $b$ and $d^{\prime}$ are not real in case (i) and $\left\{\left(\begin{array}{c}a^{\prime} \\ 1\end{array}\right),\left(\begin{array}{l}b^{\prime} \\ b\end{array}\right),\left(\begin{array}{l}c^{\prime} \\ c\end{array}\right),\left(\begin{array}{l}d^{\prime} \\ d\end{array}\right)\right\}$ is linearly independent over the real numbers in case (ii).

Proposition 2.2 leads to an improvement of 1.1 and the result in [2] as follows:

THEOREM 2.3. Let $\Gamma \backslash C^{2}$ be a compact complete locally affine complex surface. Then (i) and (ii) of Theorem 1.1 hold; and (iii) $t$, which is the index of $\Gamma_{0}$ in $\Gamma$, can take only the values $1,2,3,4$, and 6 .

Proof. We refer to the proof of Theorem 4.1 in [2] where it is shown that $t=1,2,3,4,5,6,8,10$, or 12 . We must eliminate the cases $t=5,8,10,12$. Now, the restriction on $t$ comes from the inequality $\varphi(t) \leqq r$, where is Euler's totient and $r$ is the rank of the additive subgroup of $C$ generated by the 12-entries of elements of $\Gamma_{0}$. The cases $t=5,8,10,12$ can occur only when $r=$ 4. By Proposition 2.2, this can occur only when $\Gamma_{0}$ is abelian and, in the notation there, $\lambda \neq 0$ and $1, b, c, d$ are linearly independent over the integers. The only element of such a $\Gamma_{0}$ having its 12and 23-entries zero is the identity. Suppose now that such a $\Gamma_{0}$ occurs as a subgroup of $\Gamma$ of index $t>1$. Let $S=\left(\begin{array}{ccc}1 & f & u \\ 0 & w & v \\ 0 & 0 & 1\end{array}\right)$ be the element of $\Gamma$ described after Theorem 1.1; then $S^{t}$ is in $\Gamma_{0}$. We have $S^{t}=\left(\begin{array}{lll}1 & 0 & u^{\prime} \\ 0 & 1 & 0 \\ 0 & 0 & 1\end{array}\right)$, where $u^{\prime}=t u+t f u /(1-w)$, since $w^{t}=1$. Hence $u^{\prime}=0$ and $f v-(w-1) u=0$. This contradicts Lemma 2.1. Thus, such a $\Gamma_{0}$ has no proper extensions to a group $\Gamma$.

3. Generators and relations. In this section, we shall use the matrix description of the generators $S, A, B, C, D$ of $\Gamma$ that we have obtained in order to delimit the relations that can occur for our fundamental groups $\Gamma$, as opposed to the general relations implied by the observation following Theorem 1.1. 
THEOREm 3.1. Let $\Gamma$ be a subgroup of $A(2, C)$ which acts properly on $C^{2}$ and has compact orbit space. Then $\Gamma$ has generators $S, A, B, C, D$ with relations among the following:

(1) If the group $\Gamma_{0}$ generated by $A, B, C, D$ is free abelian, we have three cases as follows:

(a) If the parameter $\lambda$ of Proposition 2.2 (ii) is zero, then the groups are given in [1].

(b) If $\lambda \neq 0$, we must have $S^{2}=D, S D=D S$, and (i) $S A S^{-1}=$ $A^{-1} D^{\alpha}, S B S^{-1}=B^{-1} D^{\beta}, S C S^{-1}=C^{-1} D^{\gamma}$, where $\alpha, \beta, \gamma$ are 0 or 1 , or (ii) $S C=C S, S A S^{-1}=A^{-1} C^{\alpha} D^{\beta}, S B S^{-1}=B^{-1} C^{\gamma} D^{\delta}$, where $\alpha, \beta, \gamma, \delta$ are all 0 or 1.

(2) If the group $\Gamma_{0}$ generated by $A, B, C, D$ has relations $A B A^{-1} B^{-1}=C^{k}, C$ and $D$ central in $\Gamma_{0}$, then the other relations may be taken as follows: $S C=C S, S D=D S, S^{t}=C^{r} D^{s}$ (where, as before, $\left.t=\left[\Gamma: \Gamma_{0}\right]\right)$, and

(a) if $t=2$ then $S A S^{-1}=A^{-1} C^{e} D^{f}$ and $S B S^{-1}=B^{-1} C^{g} D^{h}$,

(b) if $t=3$ then $S A S^{-1}=B C^{e} D^{f}$ and $S B S^{-1}=A^{-1} B^{-1} C^{g} D^{h}$,

(c) if $t=4$ then $S A S^{-1}=B C^{e} D^{f}$ and $S B S^{-1}=A^{-1} C^{g} D^{h}$, and

(d) if $t=6$ then $S A S^{-1}=B C^{e} D^{f}$ and $S B S^{-1}=A^{-1} B C^{g} D^{h}$.

Here $r$ may be chosen from among $0,1,2, \cdots, t-1$ and $s$ may be chosen from among $0,1 \cdots, r-1$, and in addition there are only finitely many choices for $e, f, g, h$.

REMARK. Not all the abstract groups above are of interest here; they must be subjected to the further condition that they act properly on $C^{2}$. This is discussed in the next section.

Proof. We know from 1.1 that we may assume $\Gamma$ is contained in the group $G$, and that $\Gamma_{0}$ is as in 2.2. Hence we take $S=$ $\left(\begin{array}{ccc}1 & x & z \\ 0 & \alpha & y \\ 0 & 0 & 1\end{array}\right)$, where $\alpha$ is a primitive th root of unity, and study the implications of the conditions that $S^{t} \in \Gamma_{0}$ and $S \Gamma_{0} S^{-1}=\Gamma_{0}$.

(1) If $\Gamma_{0}$ is abelian, and (a) the parameter $\lambda$ is zero, then the elements of $\Gamma$ are hermitian, and this case is fully discussed in [1]. If $\Gamma_{0}$ is abelian and $\lambda \neq 0$, notice that since $S=\left(\begin{array}{lll}1 & x & z \\ 0 & \alpha & y \\ 0 & 0 & 1\end{array}\right), S^{-1}=$ $\left(\begin{array}{ccc}1 & -\alpha^{-1} x & \alpha^{-1} x y-z \\ 0 & \alpha^{-1} & -\alpha^{-1} y \\ 0 & 0 & 1\end{array}\right)$ and $S\left(\begin{array}{ccc}1 & u & w \\ 0 & 1 & v \\ 0 & 0 & 1\end{array}\right) S^{-1}=\left(\begin{array}{ccc}1 & \alpha^{-1} u & -\alpha^{-1} u y+w+v x \\ 0 & 1 & \alpha v \\ 0 & 0 & 1\end{array}\right)$, we must have $\alpha=\alpha^{-1}=-1$ if $S \Gamma_{0} S^{-1}=\Gamma_{0}$ (because elements of $\Gamma_{0}$ have the property that their 12-entries equal their 23-entries multiplied by $\lambda$ ).

Next notice that 2.2 says that when the rank of the additive 
subgroup of $C$ generated by $1, b, c, d$ is 4 , the group $\Gamma_{0}$ admits no proper extensions. Also, the rank of this group cannot be 1, for then $\Gamma_{0} \mid C^{2}$ would not be compact (see [2], §3). We are therefore reduced to the following cases for an abelian $\Gamma_{0}$ :
(i) $A=\left(\begin{array}{lll}1 & \lambda & 0 \\ 0 & 1 & 1 \\ 0 & 0 & 1\end{array}\right), B=\left(\begin{array}{ccc}1 & \lambda b & b^{\prime} \\ 0 & 1 & b \\ 0 & 0 & 1\end{array}\right), C=\left(\begin{array}{ccc}1 & \lambda c & b^{\prime} \\ 0 & 1 & c \\ 0 & 0 & 1\end{array}\right), D=\left(\begin{array}{ccc}1 & 0 & d \\ 0 & 1 & 0 \\ 0 & 0 & 1\end{array}\right)$,
(ii) $A=\left(\begin{array}{lll}1 & \lambda & 0 \\ 0 & 1 & 1 \\ 0 & 0 & 1\end{array}\right), B=\left(\begin{array}{ccc}1 & \lambda b & b^{\prime} \\ 0 & 1 & b \\ 0 & 0 & 1\end{array}\right), C=\left(\begin{array}{lll}1 & 0 & c \\ 0 & 1 & 0 \\ 0 & 0 & 1\end{array}\right), D=\left(\begin{array}{lll}1 & 0 & d \\ 0 & 1 & 0 \\ 0 & 0 & 1\end{array}\right)$,

with $\lambda \neq 0$ and $1, b, c$ linearly independent over $Z$ in case (a) and $1, b$ linearly independent over $\boldsymbol{Z}$ in case (b). Also, it has been determined that $S=\left(\begin{array}{ccc}1 & x & z \\ 0 & -1 & y \\ 0 & 0 & 1\end{array}\right)$.

In case (b), we automatically have $S D=D S$; furthermore $S A S^{-1}=$ $\left(\begin{array}{ccc}1 & -\lambda & \lambda y+x \\ 0 & 1 & -1 \\ 0 & 0 & 1\end{array}\right), S B S^{-1}=\left(\begin{array}{ccc}1 & -\lambda b & \lambda b y+b^{\prime}+b x \\ 0 & 1 & -b \\ 0 & 0 & 1\end{array}\right)$, and

$$
S C S^{-1}=\left(\begin{array}{ccc}
1 & -\lambda c & \lambda c y+c^{\prime}+c x \\
0 & 1 & -c \\
0 & 0 & 1
\end{array}\right)
$$

Knowing that $S \Gamma_{0} S^{-1}=\Gamma_{0}$, we must therefore have $S A S^{-1}=A^{-1} D^{\alpha}$, $S B S^{-1}=B^{-1} D^{\beta}, S C S^{-1}=C^{-1} D^{r}$, as we see from a glance at the 23entries of the above matrix. Now, the group $\Gamma$ is generated by $S$, $A D^{m}, B D^{p}, C D^{q}, D$ as well as by $S, A, B, C, D$; seeing that $S A D^{m} S^{-1}=$ $A^{-1} D^{\alpha} D^{m}=A^{-1} D^{-m} D^{\alpha+2 m}$, etc., we may assume that $\alpha, \beta, \gamma$ above are reduced independently modulo 2 .

Now impose the condition $S^{2} \in \Gamma_{0}$. Since $S^{2}=\left(\begin{array}{ccc}1 & 0 & 2 z+x y \\ 0 & 1 & 0 \\ 0 & 0 & 1\end{array}\right)$, we must have $S^{2}=D^{k}$ for some $k . \quad \Gamma$ is generated by $S D^{m}, A, B, C, D$ as well as by $S, A, B, C, D$, and $\left(S D^{m}\right)^{2}=D^{k+2 m}$, so $k$ can be reduced modulo 2 also. If $k=0$ then $S^{2}=\mathrm{id}$, so $2 z+x y=0$, in which case $S \in \Gamma$ has a fixed point (see 2.1). Hence we may take $k=1$ for purposes of this paper. This takes care of 3.1.1.b. (i).

Turning now to case (ii) where $\Gamma_{0}$ is abelian and $\lambda \neq 0$, we first impose the condition that $S^{2} \in \Gamma_{0}$. This being the case, we must have $S^{2}=C^{a} D^{b}$. Replacing $S$ by $S_{1}=S C^{m} D^{n}$, we have $S_{1}^{2}=S^{2} C^{2 m} D^{2 n}=$ $C^{a+2 m} D^{b+2 n}$, since $S C=C S$ and $S D=D S$. Hence $a$ and $b$ may be chosen to be 0 or 1 . If $a=b=0$ then $S$ again has a fixed point. The other three cases give the same group, so we may take $S^{2}=D$.

The other relations to contend with are those giving the form of $S A S^{-1}$ and $S B S^{-1}$. A glance at 23-entries shows that we must have $S A S^{-1}=A^{-1} C^{a_{1}} D^{a_{2}}$ and $S B S^{-1}=B^{-1} C^{b_{1}} D^{b_{2}}$, and replacement of 
the generators $S, A, B, C, D$ by $S, A C^{m} D^{n}, B C^{p} D^{q}, C, D$ shows that $a_{1}$, $a_{2}, b_{1}, b_{2}$ may be independently taken to be 0 or 1 . The case where $\Gamma_{0}$ is abelian is now taken care of.

When $\Gamma_{0}$ is nonabelian, we have $A=\left(\begin{array}{lll}1 & a & 0 \\ 0 & 1 & 1 \\ 0 & 0 & 1\end{array}\right), B=\left(\begin{array}{cccc}1 & a b & -k & 0 \\ 0 & 1 & b \\ 0 & 0 & 1\end{array}\right)$, $C=\left(\begin{array}{lll}1 & 0 & 1 \\ 0 & 1 & 0 \\ 0 & 0 & 1\end{array}\right)$, and $D=\left(\begin{array}{lll}1 & 0 & d \\ 0 & 1 & 0 \\ 0 & 0 & 1\end{array}\right)$, so that $A B=B A C^{k}$ and $C$ and $D$ are central in $\Gamma_{0}$. Then $\Gamma_{0}$ consists of all words $A^{a} B^{b} C^{c} D^{d}$. Two such words are equal only when the corresponding exponents are equal, and

$$
\left(A^{a} B^{b} C^{c} D^{d}\right)\left(A^{r} B^{s} C^{t} D^{u}\right)=A^{a+r} B^{b+s} C^{c+t-r b k} D^{t+u} .
$$

The elements $A_{1}=A^{m} B^{n} C^{e} D^{f}, B_{1}=A^{p} B^{q} C^{g} D^{h}, C_{1}=C^{m q-n p}$, and $D_{1}=$ $C^{c} D^{ \pm 1}$ of $\Gamma_{0}$ may be seen to generate $\Gamma_{0}$ provided $m q-n p= \pm 1$, and then they satisfy the same relations as $A, B, C, D$. If we were to choose these different generators for $\Gamma_{0}$, we could, according to 2.2 , still conjugate them back to the canonical form above, but with possibly different values of the parameters $a, b$, and $d$.

Now look for $S=\left(\begin{array}{lll}1 & x & z \\ 0 & \alpha & y \\ 0 & 0 & 1\end{array}\right)$ such that $S^{t} \in \Gamma_{0}\left(\right.$ i.e., $\left.\alpha^{t}=1\right)$ for $t=$ $2,3,4,6$, and $S \Gamma_{0} S^{-1}=\Gamma_{0}$.

We must have $S^{t}=C^{r} D^{s}$. Replacing $S$ by $S_{1}=S C^{m} D^{n}$, we get $S_{1}^{t}=S^{t} C^{m t} D^{n t}=C^{r+m t} D^{s+n t}$, so that we may take $r$ and $s$ to be reduced modulo $t$. Then replacing $C$ by $C D^{p}=C_{1}$, we have $S^{t}=C^{r} D^{s}=$ $\left(\mathrm{CD}^{p}\right)^{r} D^{s-p r}=C_{1}^{r} D^{s-p r}$, so that we may further take $s$ to be reduced modulo $r$.

Next impose the condition $S \Gamma_{0} S^{-1}=\Gamma_{0}$. We automatically have $S C=C S$ and $S D=D S$, so this condition is the same as saying that $S A S^{-1}=A^{m} B^{n} C^{e} D^{f}$, and $S B S^{-1}=A^{p} B^{q} C^{g} D^{h}$, with $m q-n p=1$. (Since $S C S^{-1}=C$ and conjugation by $S$ is an automorphism of $\Gamma_{0}, m q-$ $n p \neq-1$.) Then $\left(\begin{array}{ll}m & n \\ p & q\end{array}\right)$ is an integer matrix whose $t$ th power is the identity $(t=2,3,4$, or 6$)$. There are lots of these; however it is well known that there is an integer matrix $\left(\begin{array}{ll}x & y \\ z & w\end{array}\right)$ with $x w-y z= \pm 1$ such that $M\left(\begin{array}{ll}x & y \\ z & w\end{array}\right)=\left(\begin{array}{ll}x & y \\ z & w\end{array}\right)\left(\begin{array}{ll}m & n \\ p & q\end{array}\right)$, where $M=\left(\begin{array}{rr}-1 & 0 \\ 0 & -1\end{array}\right)$ if $t=2$, $\left(\begin{array}{rr}0 & 1 \\ -1 & 0\end{array}\right)$ if $t=4,\left(\begin{array}{rr}0 & 1 \\ -1 & -1\end{array}\right)$ if $t=3$, and $\left(\begin{array}{rr}0 & 1 \\ -1 & 1\end{array}\right)$ if $t=6$. Let $S_{1}=$ $S, A_{1}=A^{x} B^{y}, B_{1}=A^{z} B^{w}, C_{1}=C^{x w-y z}, D_{1}=D$. Then, as noted above, these generate $\Gamma$, and $A_{1}, B_{1}, C_{1}, D$, satisfy the same relation as $A$, $B, C, D$. Now, however, we have

$$
\begin{aligned}
S A_{1} S^{-1} & =S A^{x} B^{y} S^{-1} \\
& =\left(S A S^{-1}\right)^{x}\left(S B S^{-1}\right)^{y} \\
& =\left(A^{m} B^{n}\right)^{x}\left(A^{p} B^{q}\right)^{y} P,
\end{aligned}
$$


where $P$ involves powers of $C$ and $D$. Modulo powers of $C$ and $D$, we have $S A_{1} S^{-1} \equiv A^{m x+p y} B^{n x+q y}$ and similarly, $S B_{1} S^{-1} \equiv A^{m z+p w} B^{n z+q w}$.

The following table then describes $S A_{1} S^{-1}$ and $S B_{1} S^{-1}$ according to $\left.] \Gamma: \Gamma_{0}\right]=t$ :

\begin{tabular}{c|c|c|c|c} 
& $t=2$ & $t=3$ & $t=4$ & $t=6$ \\
\hline$S A_{1} S^{-1}$ & $A_{1}^{-1} C_{1}^{e_{1}} D_{1}^{f_{1}}$ & $B_{1} C_{1}^{e_{1}} D_{1}^{f_{1}}$ & $B_{1} C_{1}^{e_{1}} D_{1}^{f_{1}}$ & $B_{1} C_{1}^{e_{1}} D_{1}^{f_{1}}$ \\
\hline$S B_{1} S^{-1}$ & $B_{1}^{-1} C_{1}^{g_{1}} D_{1}^{h_{1}}$ & $A_{1}^{-1} B_{1}^{-1} C_{1}^{q_{1}} D_{1}^{h_{1}}$ & $A_{1}^{-1} C_{1}^{g_{1}} D_{1}^{h_{1}}$ & $A_{1}^{-1} B_{1} C_{1}^{g_{1}} D_{1}^{h_{1}}$ \\
\hline
\end{tabular}

We may thus normalize conjugation of $A$ and $B$ by $S$ as above; the other relations among $S, A, B, C, D$ remain as before.

We next wish to normalize powers of $C$ and $D$ that occur when $A$ and $B$ are conjugated by $S$. To this end, assume that $S A S^{-1}=$ $A^{m} B^{n} C^{e} D^{f}$ and $S B S^{-1}=A^{p} B^{q} C^{g} D^{h}$. Replace the generators $S, A, B$, $C, D$ by $S, A_{1}=A C^{x} D^{y}, B_{1}=B C^{z} D^{w}, C$, and $D$. All relations remain the same except conjugation of $A_{1}$ and $B_{1}$ by $S$ where we get $S A_{1} S^{-1}=$ $A^{m} B^{n} C^{e+x} D^{f+y}$ and $S B_{1} S^{-1}=A^{p} B^{q} C^{g+z} D^{h+w}$, or,

$$
S A_{1} S^{-1}=\left(A C^{x} D^{y}\right)^{m}\left(B C^{z} D^{w}\right)^{n} C^{e+x-m x-n z} D^{f+y-m y-n w}
$$

and

$$
S B_{1} S^{-1}=\left(A C^{x} D^{y}\right)^{p}\left(B C^{z} D^{w}\right)^{q} C^{g+z-p x-q z} D^{h+w-p y-q w} .
$$

We may rewrite this as $S A_{1} S^{-1}=A_{1}^{m} B_{1}^{n} C^{e_{1}} D^{f_{1}}$ and $S B_{1} S^{-1}=A_{1}^{p} B_{1}^{q} C^{g_{1}} D^{h_{1}}$, where

$$
\begin{aligned}
\left(\begin{array}{ll}
e_{1} & f_{1} \\
g_{1} & h_{1}
\end{array}\right) & =\left(\begin{array}{ll}
e & f \\
g & h
\end{array}\right)+\left(\begin{array}{cc}
x & y \\
z & w
\end{array}\right)-\left(\begin{array}{cc}
m & n \\
p & q
\end{array}\right)\left(\begin{array}{ll}
x & y \\
z & w
\end{array}\right) \\
& =\left(\begin{array}{ll}
e & f \\
g & h
\end{array}\right)-\left(\begin{array}{cc}
m-1 & n \\
p & q-1
\end{array}\right)\left(\begin{array}{cc}
x & y \\
z & w
\end{array}\right) .
\end{aligned}
$$

We are thus led to the matrix congruence relation $\left(\begin{array}{ll}e & f \\ g & h\end{array}\right) \equiv\left(\begin{array}{ll}e_{1} & f_{1} \\ g_{1} & h_{1}\end{array}\right)$ provided their difference is a multiple of $\left(\begin{array}{c}m-1 \\ p\end{array}-1-1\right)$. Viewing the set $M$ of all $2 \times 2$ integer matrices as a $Z$-module (under matrix addition, of course), we see that the set of all multiples of

$$
\left(\begin{array}{cc}
m-1 & n \\
p & q-1
\end{array}\right)
$$

is a submodule $M_{1}$, and the equivalence classes above are the same as the elements of $M / M_{1}$. One may easily verify that the matrices $\left(\begin{array}{cc}m-1 & n \\ p & q-1\end{array}\right)$ that arise when $t=2,3,4$, and 6 are nonsingular. Hence $M$ and $M_{1}$ have the same rank, which means that $M / M_{1}$ is 
finite. In other words, there are only finitely many choices of $e, f, g$, and $h$ that give nonisomorphic groups.

This concludes the proof of Theorem 3.1.

4. Topological results. In this section, we discuss certain results having to do with transformation groups which are of use in the further study of the groups of 3.1.

Recall that a group $\Gamma$ of homeomorphisms of a space $X$ is said to act properly on $X$ if each $x \in X$ has a neighborhood $U$ such that $\gamma U \cap U=\varnothing$ for each $\gamma \neq \mathrm{id}$. in $\Gamma$. Notice that if $\Gamma$ acts properly then $\Gamma$ acts freely (i.e., no element of $\Gamma$ except the identity has any fixed point). A subset $F$ of the space $X$ will be called a fundamental domain for $\Gamma$ if $F$ contains exactly one point of each $\Gamma$-orbit.

Proposition 4.1. Suppose that $\Gamma$ is a freely-acting group of homeomorphisms of a space $X$, and that $\Gamma$ has a subgroup $\Gamma_{0}$ of finite index that acts properly. Let $F$ be a fundamental domain for $\Gamma_{0}$ and assume that there is an open set $G \supset F$ such that the cardinality of $\left\{\beta \in \Gamma_{0} \mid \beta G \cap G \neq \varnothing\right\}$ is finite. Then $\Gamma$ acts properly.

The proof of 4.1 will be omitted, as it is not part of the main point of the present article.

It is not difficult to see that the groups $\Gamma_{0}$ of 2.3 have as fundamental domain a product of two half-open parallelograms spanned by the translation parts of the generators $A, B, C, D$. An open set $G$ of the kind mentioned above in 4.1 is then readily obtained. As we have already remarked, the groups $\Gamma_{0}$ act properly on $C^{2}$. Hence, to prove that the groups of 3.1 act properly, it suffices to prove that they act freely. It is easily verified that the groups $\Gamma_{0}$ of 2.3 act freely; this being the case, there is a convenient abstract condition that insures that $\Gamma$ acts freely, as follows.

Proposition 4.2. Let $\Gamma$ be group of affine motions having a subgroup $\Gamma_{0}$ of finite index which acts freely. Then $\Gamma$ acts freely if and only if $\Gamma$ has no elements of finite order.

The proof of 4.2 will also be omitted.

5. Conclusion. In light of $\S 4$, we may conclude our classification by seeing which of the groups of Theorem 3.1 are torsionfree and then checking to see that these can indeed be embedded in the group $G$ of Theorem 1.1. It will be seen that the task of carrying out these two steps is quite lengthy (though elementary), so that many results will be given without proof. However, a method of arriving at them will be described completely and application in some 
representative cases will be given.

First we take care of the case where $\Gamma_{0}$ is abelian.

THEOREM 5.1. The following groups (and only those) from (3.1.1b) are torsion-free:

(i) $S^{2}=D, S D=D S, S A S^{-1}=A^{-1}, S B S^{-1}=B^{-1}, S C S^{-1}=C^{-1}$;

(ii) $S^{2}=D, S D=D S, S C=C S, S A S^{-1}$

$$
=A^{-1} C^{\alpha} D^{\beta}, S B S^{-1}=B^{-1} C^{r} D^{\delta} \text {, }
$$

where (1) $\alpha=\beta=\gamma=\delta=0$, (2) $\alpha=1, \beta=\gamma=\delta=0$, (3) $\gamma=1, \alpha=$ $\beta=\delta=0$, (4) $\alpha=\beta=1, \gamma=\delta=1$, (5) $\alpha=\gamma=1, \beta=\delta=\delta=0$, (6) $\alpha=$ $\beta=0, \gamma=\delta=1$ and (7) $\alpha=\beta=\gamma=\delta=1$. Furthermore, each of these can be embedded in the group $G$ of 1.1 .

Proof. We restrict attention to case (i). If $X \in \Gamma$ has finite order, then $X \in S \Gamma_{0}$ and $X^{2}=1$. Writing $X=S A^{m} B^{n} C^{p} D^{q}$, we have $X^{2}=D^{m \alpha+n \beta+p \gamma+2 q+1}$. Clearly the exponent is always nonzero if and only if $\alpha=\beta=\gamma=0$ (recall that $\alpha, \beta, \gamma$ are 0 or 1 ). Hence only one case gives a torsion-free group.

As for the embedding problem, recall that $S, A, B, C, D$ have the form $\left(\begin{array}{rrr}1 & x & z \\ 0 & -1 & y \\ 0 & 0 & 1\end{array}\right),\left(\begin{array}{ccc}1 & a & a^{\prime} \\ 0 & 1 & 1 \\ 0 & 0 & 1\end{array}\right),\left(\begin{array}{ccc}1 & a b & b^{\prime} \\ 0 & 1 & b \\ 0 & 0 & 1\end{array}\right),\left(\begin{array}{ccc}1 & a c & c^{\prime} \\ 0 & 1 & c \\ 0 & 0 & 1\end{array}\right)$ and $\left(\begin{array}{ccc}1 & a d & d^{\prime} \\ 0 & 1 & d \\ 1 & 0 & 1\end{array}\right)$ respectively. Then $S^{2}=\left(\begin{array}{ccc}1 & 0 & x y+2 z \\ 0 & 1 & 0 \\ 0 & 0 & 1\end{array}\right)=D$ is easily satisfied and $S D=$ $D S$ is automatic. The other three relations lead to four equations involving the ten parameters $x, y, z, a, a^{\prime}, b, b^{\prime}, c, c, d$ and $d^{\prime}$ and there are many solutions.

From here on, we deal only with the cases where $\Gamma_{0}$ is nonabelian. Therefore, $\Gamma$ has generators $S, A, B, C, D$ and relations as follows: $A B=B A C^{k}$ with $k \geqq 1 ; C$ and $D$ central in $\Gamma ; S$ has order $t=2,3$, 4 or 6 modulo $\Gamma_{0}$ and $S^{t}=C^{r} D^{s}$, where $r$ and $s$ are reduced modulo $t$, and $s$ is further reduced modulo $r$; finally, we have

(a) if $t=2, S A S^{-1}=A^{-1} C^{e} D^{f}$ and $S B S^{-1}=B^{-1} C^{g} D^{h}$,

(b) if $t=3, S A S^{-1}=B C^{e} D^{f}$ and $S B S^{-1}=A^{-1} B^{-1} C^{g} D^{h}$,

(c) if $t=4, S A S^{-1}=B C^{e} D^{f}$ and $S B S^{-1}=A^{-1} C^{g} D^{h}$, and

(d) if $t=6, S A S^{-1}=B C^{e} D^{f}$ and $S B S^{-1}=A^{-1} B C^{g} D^{h}$.

Recall from the proof of 3.1 that for each $t$, only finitely many choices of $e, f, g, h$ give distinct groups. More precisely, $\left(\begin{array}{ll}e & f \\ g & h\end{array}\right) \sim$ $\left(\begin{array}{ll}e^{\prime} & f^{\prime} \\ g^{\prime} & h^{\prime}\end{array}\right)$ provided $\left(\begin{array}{ll}e & f \\ g & h\end{array}\right)-\left(\begin{array}{ll}e^{\prime} & f^{\prime} \\ g^{\prime} & f^{\prime}\end{array}\right)=T\left(\begin{array}{ll}x & y \\ z & w\end{array}\right)$ for some $x, y, z, w \in Z$, where $T=\left(\begin{array}{rr}-2 & 0 \\ 0 & -2\end{array}\right)$ if $t=2,\left(\begin{array}{lr}-1 & 1 \\ -1 & -2\end{array}\right)$ if $t=3,\left(\begin{array}{rr}-1 & 1 \\ -1 & -1\end{array}\right)$ if $t=4$, 
and $\left(\begin{array}{cc}-1 & 1 \\ -1 & 0\end{array}\right)$ if $t=6$. Accordingly, we have the following possibilities for $e, f, g, h$ :

(a) if $t=2, e, f, g, h$ independently chosen to be 0 or 1 ;

(b) if $t=3, g=h=0$ and $e, f$ chosen to be 0,1 or 2;

(c) if $t=4, g=h=0$ and $e, f$ chosen to be 0 or 1 ;

(d) if $t=6, e=f=g=h=0$. Therefore, when $\Gamma_{0}$ is nonabelian, there are 133 cases to study (ignoring the cases where $r=s=0$ and the infinite dependence on $k$ ).

To determine which of these groups are torsion-free, we must check each coset $S^{i} \Gamma_{0}, i=1, \cdots, t-1$, in each group for elements of finite order. All together, there are 330 cosets to be studied. In each coset we take a general element $X=S^{i} A^{m} B^{n} C^{p} D^{q}$ whose order $u$, if finite, is easily determined. Then $X^{u}$ is always of the form $C^{v} D^{w}$, where $v$ and $w$ depend on $k, m, n, p$ and $q$ (and $i$ and $\Gamma$, also, of course), so $X^{u}=1$ if and only if $v$ and $w$ can be made zero by some choice of integers $k, m, n, p$ and $q$.

Computation gives the following answers for $v$ and $w$ :

(a) if $t=2$ and $X \in S \Gamma_{0}$, then $v=m n k+m e+n g+2 p+r$ and $w=m f+n h+2 q+s$

(b) if $t=3$ and $X \in S \Gamma_{0}$, then $v=(1 / 2) k(m+n)(m+n+1)+$ $2 m e-n e+3 p+r$ and $w=2 m f-n f+3 q+s$;

if $X \in S^{2} \Gamma_{0}$, then $v=(1 / 2) k m(m+1)+(1 / 2) k n(n+1)+2 m e-n e+$ $3 p+2 r$ and $w=2 m f-n f+3 q+2 s$;

(c) if $\mathrm{t}=4$ and $X \in S \Gamma_{0}$, then $v=k(m+n)^{2}+2 m e-2 n e+4 p+$ $r$ and $w=2 m f-2 n f+4 q+s$;

if $X \in S^{2} \Gamma_{0}$, then $v=k m n+m e-n e+2 p+r$ and $w=m f-$ $n f+2 q+s$

if $X \in S^{3} \Gamma_{0}$, then $v=-k(m+n)^{2}+2 m e-2 n e+4 p+3 r$ and $w=$ $2 m f-2 n f+4 q+s$;

(d) if $t=6$ and $X \in S \Gamma_{0}$, then $v=3 k(m+n)^{2}-3 n k-3 m k+$ $6 p+r$ and $w=6 q+s$;

if $X \in S^{2} \Gamma_{0}$, then $v=2 k m n+(1 / 2) k m(m-1)+(1 / 2) k n(n+1)-$ $2 n k-m k+3 p+r$ and $w=3 q+s$;

if $X \in S^{3} \Gamma_{0}$, then $v=m n k-m k-n k+2 p+r$ and $w=2 q+s$;

if $X \in S^{4} \Gamma_{0}$, then $v=-(1 / 2) k(m+n)(m+n+3)+3 p+2 r$ and $w=3 q+2 s$

if $X \in S^{5} \Gamma_{0}$, then $v=-3 k\left(m^{2}+m+n^{2}+n\right)+6 p+5 r$ and $w=$ $6 q+5 s$.

From this, some cases are easily seen to be torsionfree. For example, when $t=3, f=0, s=1$, or when $t=6$ and $s=1$, no $X \in$ $\Gamma$ has finite order since $3 q+1,3 q+2,6 q+1$, etc. are never zero. Other cases require a bit more; for example, consider the case $t=3$, $e=f=1, r=0, s=1$. Then $S \Gamma_{0}$ has an element of finite order if 
and only if $(1 / 2) k(m+n)(m+n+1)+2 m-n+3 p=0$ and $2 m-$ $n+3 q+1=0$, and $S^{2} \Gamma_{0}$ has an element of finite order if and only if $(1 / 2) \mathrm{km}(m+1)+(1 / 2) k n(n+1)+2 m-n+3 p=0$ and $2 m-n+$ $3 q+2=0$, for some $k, m, n, p, q$. The first system is equivalent to finding $(1 / 2) k(m+n)(m+n+1)-3 q+3 p-1=0$, which can happen if and only if $k \equiv 1(\bmod 3)$, and the second system is equivalent to $(1 / 2) k m(m+1)+(1 / 2) k n(n+1)+3 p-3 q-2=0$, which can happen if and only if $k \equiv 1$ or $2(\bmod 3)$. Therefore the group in this case is torsion-free if and only if $k \equiv 0(\bmod 3)$.

The complete search for elements of finite order gives the following result.

THEOREM 5.2. The following groups (and only those) from 3.1 .2 are torsion-free.

(a) (when $t=2$ ) The following cases are torsion-free without restriction on $k: r=0, s=1$ and: $e=f=g=h=0$, or $e=1$ and $f=g=h=0$, or $g=1$ and $e=f=h=0$, or $e=g=1$ and $f=$ $h=0$, or $e=f=g=h=1 ; r=1, s=0$ and: $f=1$ and $e=g=$ $h=0$, or $h=1$ and $e=f=g=0$, or $e=f=1$ and $g=h=0$, or $e=f=0$ and $g=h=1$, or $e=0$ and $f=g=h=1$, or $e=f=$ $h=1$ and $g=0$. When $k$ is even, the following are torsion-free: $r=1, s=0$ and: $e=f=g=h=0$, or $e=g=0$ and $f=h=1$, or $e=f=g=h=1$. When $k$ is odd, the following are torsion-free: $r=0, s=1$ and: $e=g=h=1$ and $f=0$, or $e=f=g=1$ and $h=0$.

(b) (when $t=3$ ) The following cases are torsion-free without restriction on $k: r=0, s=1$ and $e=f=0$, or $e=1$ and $f=0$, or $e=2$ and $f=0 ; r=0, s=2$ and $e=f=0$, or $e=1$ and $f=0$, or $e=2$ and $f=0 ; r=2, s=1$ and $e=f=0$, or $e=1$ and $f=0$, or $e=2$ and $f=0$. When $k \equiv 0(\bmod 3)$, the following cases are torsionfree: $r=0, s=1$ and $e=f=1$, or $e=2$ and $f=1$, or $e=$ 1 and $f=2 ; r=0, s=2$ and $e=f=1$, or $e=2$ and $f=1$, or $e=$ 1 and $f=2 ; r=1, s=0$ and $e=f=0$, or $e=0$ and $f=1$, or $e=$ $f=1$, or $e=2$ and $f=1$, or $e=1$ and $f=2$, or $e=f=2 ; r=$ $2, s=0$ and $e=f=1$, or $e=0$ and $f=1$, or $e=f=1$, or $e=2$ and $f=1$, or $e=1$ and $f=2$; or $e=f=2 ; r=2, s=1$ and $e=$ $f=1$. When $k \equiv 0$ or $1(\bmod 3)$, the following cases are torsion-free: $r=0, s=2$ and $e=f=2 ; r=1, s=0$ and $e=0, f=2$. When $k \equiv 0$ or $2(\bmod 3)$, the following are torsion-free: $r=0, s=1$ and $e=f=2 ; r=2, s=0$ and $e=0, f=2 ; r=2, s=1$ and $e=f=0$. When $k \equiv 1$ or $2(\bmod 3)$, the following is torsion-free: $r=0, s=2$, $e=0, f=2$.

(c) (when $t=4$ ) The following are torsion-free without restriction on $k: r=0, s=1$ and $e=f=0$, or $e=1$ and $f=0 ; r=0, s=$ 
2 and $e=f=0$, or $e=1$ and $f=0 ; r=0, s=3$ and $e=f=0$, or $e=1$ and $f=0 ; r=2, s=1$ and $e=f=0$, or $e=1$ and $f=0$; $r=3, s=1$ and $e=f=0$, or $e=0$ and $f=1$, or $e=1$ and $f=0$; $r=3, s=2$ and $e=f=0$ or $e=1$ and $f=0$. When $k$ is even, the following are torsion-free: $r=0, s=1, e=f=1 ; r=0, s=3, e=$ $f=1 ; r=1, s=0, e=f=0$ or $e=0$ and $f=1$, or $e=f=1 ; r=$ $2, s=1, e=f=1 ; r=3, s=0, e=f=0$, or $e=0$ and $f=1$, or $e=f=1 ; r=3, s=2, e=0$ and $f=1$, or $e=f=1$.

(d) (when $t=6$ ) The following are torsion-free regardless of the value of $k: r=0, s=1 ; r=0, s=5 ; r=2, s=1 ; r=3, s=1$; $r=4, s=1 ; r=5, s=1$. When $k$ is even, the following are torsionfree: $r=3, s=2 ; r=5, s=2 ; r=5, s=4$. When $k \equiv 0(\bmod 3)$, the following are torsion-free: $r=4, s=3 ; r=5, s=3$. When $k \equiv$ $0(\bmod 6)$, the following are torsion-free: $r=1, s=0 ; r=5, s=0$.

We shall now see that all the groups above can be embedded in the group $G$ of 1.1. In fact, we have the following result.

THEOREM 5.3. All the groups of 3.1 .2 can be embedded in the group $G$ of Theorem 1.1 .

Proof. We take $S=\left(\begin{array}{ccc}1 & x & z \\ 0 & w & y \\ 0 & 0 & 1\end{array}\right), A=\left(\begin{array}{lll}1 & a & 0 \\ 0 & 1 & 1 \\ 0 & 0 & 1\end{array}\right),\left(\begin{array}{llll}1 & a b-k & 0 \\ 0 & 1 & b \\ 0 & 0 & 1\end{array}\right)=B$, $C=\left(\begin{array}{lll}1 & 0 & 1 \\ 0 & 1 & 0 \\ 0 & 0 & 1\end{array}\right)$, and $D=\left(\begin{array}{lll}1 & 0 & d \\ 0 & 1 & 0 \\ 0 & 0 & 1\end{array}\right)$, where $w$ is a primitive $t$ th root of unity, and try to choose the parameters $x, y, z, a, b, d$ so that the relations of 3.1.2 hold: $A B=B A C^{k}, C$ and $D$ central in $\Gamma, S^{t}=C^{r} D^{s}$, and $S A S^{-1}$ and $S B S^{-1}$ as given in 3.1.2 as a function of $t$. The first two of these are consequences of 2.2 and the shape of $S$. The remaining relations are treated separately for $t=2,3,4,6$. For purposes of calculation, note that

$$
S^{t}=\left(\begin{array}{ccc}
1 & 0 & t\left(z-\frac{x y}{w-1}\right) \\
0 & 1 & 0 \\
0 & 0 & 1
\end{array}\right), S^{-1}=\left(\begin{array}{rrr}
1 & x-z-x y \\
0 & -1 & y \\
0 & 0 & 1
\end{array}\right), A^{-1}=\left(\begin{array}{rrr}
1 & -a & a \\
0 & 1 & -1 \\
0 & 0 & 1
\end{array}\right),
$$

and $B^{-1}=\left(\begin{array}{ccc}1 & -a b k & b(a b-k) \\ 0 & 1 & -b \\ 0 & 0 & 1\end{array}\right)$.

In case $t=2$, the relations lead to the following system of equations: 


$$
\begin{aligned}
& 2\left(z-\frac{x y}{-2}\right)=r+d s \\
& a y+x=a+e+f d \\
& y(a b-k)+b x=b(a b-k)+g+h d .
\end{aligned}
$$

These can be solved uniquely for $x, y$ and $z$, so that when $t=2$, there is a unique $S$ for each value of $a, b, d$ so that the required relations hold.

The other cases $t=3,4,6$ are slightly different in that the parameters $a, b$ are uniquely determined and then for each $d, S$ is uniquely determined. To illustrate, in case $t=3$, the relations in question lead to the following system:

$$
\begin{gathered}
3\left(z-\frac{x y}{w-1}\right)=r+d s \\
w^{-1} a=a b-k \\
w=b \\
-w^{-1} a y+x=e+f d \\
w^{-1}(a b-k)=-a b+k-a \\
b w=-b-1 \\
-w^{-1} y(a b-k)+b x=b(a b-k)+g+h d+a b+a .
\end{gathered}
$$

In view of (3), (6) is automatic. Solve (2) for a and then (5) is automatic. Then solve (4) and (7) for $y$ and $x$, and after that, solve (1) for $z$.

We now have in Theorems 5.1 and 5.2, together with the groups in [1], a complete list of the fundamental groups of compact complete locally affine complex surfaces. It should be noted in closing that some of the groups in this list may be isomorphic; however, it does not seem that the list of isomorphism classes is markedly shorter than the list above.

\section{REFERENCES}

1. L. Auslander, Four dimensional locally Hermitian manifolds, Trans. Amer. Math. Soc., 84 (1957), 379-391.

2. J. P. Fillmore and J. Scheuneman, Fundamental groups of compact complete locally affine complex surfaces, Pacific J. Math., 44 (1973), 487-496.

Received August 12, 1973.

Present address: 24 S. Dutcher St.

Irvington, NY 10533 


\section{PACIFIC JOURNAL OF MATHEMATICS}

EDITORS

RICHARD ARens (Managing Editor)

University of California

Los Angeles, California 90024

\section{R. A. Beaumont}

University of Washington

Seattle. Washington 98105

\section{J. DugundjI}

Department of Mathematics University of Southern California Los Angeles, California 90007

D. Gilbarg and J. Milgram

Stanford University

Stanford. California 94305

\section{ASSOCIATE EDITORS}
E. F. BECKENBACH
B. H. NEUMANN
F. WOLF
K. Yoshida

\section{SUPPORTING INSTITUTIONS}

\author{
UNIVERSITY OF BRITISH COLUMBIA \\ CALIFORNIA INSTITUTE OF TECHNOLOGY \\ UNIVERSITY OF CALIFORNIA \\ MONTANA STATE UNIVERSITY \\ UNIVERSITY OF NEVADA \\ NEW MEXICO STATE UNIVERSITY \\ OREGON STATE UNIVERSITY \\ UNIVERSITY OF OREGON \\ OSAKA UNIVERSITY
}

\author{
UNIVERSITY OF SOUTHERN CALIFORNIA \\ STANFORD UNIVERSITY \\ UNIVERSITY OF TOKYO \\ UNIVERSITY OF UTAH \\ WASHINGTON STATE UNIVERSITY \\ UNIVERSITY OF WASHINGTON

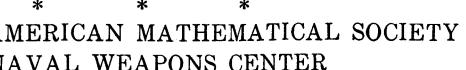

The Supporting Institutions listed above contribute to the cost of publication of this Journal, but they are not owners or publishers and have no responsibility for its content or policies.

Mathematical papers intended for publication in the Pacific Journal of Mathematics should be in typed form or offset-reproduced, (not dittoed), double spaced with large margins. Underline Greek letters in red, German in green, and script in blue. The first paragraph or two must be capable of being used separately as a synopsis of the entire paper. Items of the bibliography should not be cited there unless absolutely necessary, in which case they must be identified by author and Journal, rather than by item number. Manuscripts, in duplicate if possible, may be sent to any one of the four editors. Please classify according to the scheme of Math. Rev. Index to Vol. 39. All other communications to the editors should be addressed to the managing editor, or Elaine Barth, University of California, Los Angeles, California, 90024.

100 reprints are provided free for each article, only if page charges have been substantially paid Additional copies may be obtained at cost in multiples of 50 .

The Pacific of Journal Mathematics is issued monthly as of January 1966. Regular subscription rate: $\$ 72.00$ a year (6 Vols., 12 issues). Special rate: $\$ 36.00$ a year to individual members of supporting institutions.

Subscriptions, orders for back numbers, and changes of address should be sent to Pacific Journal of Mathematics, 103 Highland Boulevard, Berkeley, California, 94708.

\section{PUBLISHED BY PACIFIC JOURNAL OF MATHEMATICS, A NON-PROFIT CORPORATION}

Printed at Kokusai Bunken Insatsusha (International Academic Printing Co., Ltd.), 270, 3-chome Totsuka-cho. Shinjuku-ku, Tokyo 160. Japan.

Copyright (C) 1973 by Pacific Journal of Mathematics Manufactured and first issued in Japan 


\section{Pacific Journal of Mathematics}

\section{Vol. 52, No. $2 \quad$ February, 1974}

Harm Bart, Spectral properties of locally holomorphic vector-valued functions .....

J. Adrian (John) Bondy and Robert Louis Hemminger, Reconstructing infinite

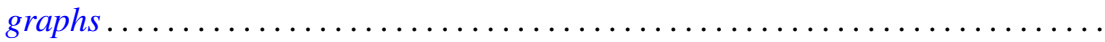

Bryan Edmund Cain and Richard J. Tondra, Biholomorphic approximation of planar

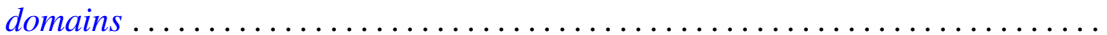

Richard Carey and Joel David Pincus, Eigenvalues of seminormal operators,

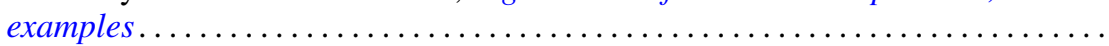

Tyrone Duncan, Absolute continuity for abstract Wiener spaces . . . . . . . . . . . . Joe Wayne Fisher and Louis Halle Rowen, An embedding of semiprime

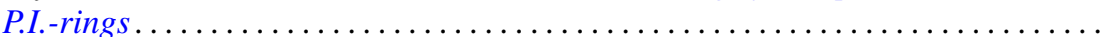

Andrew S. Geue, Precompact and collectively semi-precompact sets of semi-precompact continuous linear operators. . . . . . . . . . . . . . . .

Charles Lemuel Hagopian, Locally homeomorphic $\lambda$ connected plane continua ..... . Darald Joe Hartfiel, A study of convex sets of stochastic matrices induced by

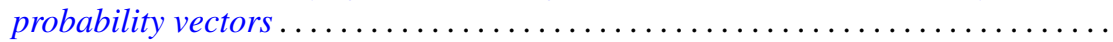

Yasunori Ishibashi, Some remarks on high order derivations $\ldots \ldots \ldots \ldots \ldots \ldots \ldots$ Donald Gordon James, Orthogonal groups of dyadic unimodular quadratic forms.

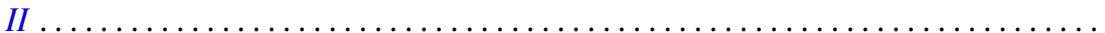

Geoffrey Thomas Jones, Projective pseudo-complemented semilattices . . . . . . . . . Darrell Conley Kent, Kelly Denis McKennon, G. Richardson and M. Schroder,

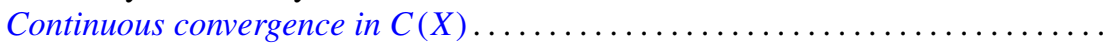

J. J. Koliha, Some convergence theorems in Banach algebras ...

Tsang Hai Kuo, Projections in the spaces of bounded linear oper

George Berry Leeman, Jr., A local estimate for typically real functions . .

475

Andrew Guy Markoe, A characterization of normal analytic spaces by the

homological codimension of the structure sheaf .........

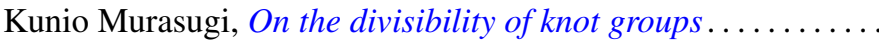

John Phillips, Perturbations of type I von Neumann algebras.

Billy E. Rhoades, Commutants of some quasi-Hausdorff matrices . .

David W. Roeder, Category theory applied to Pontryagin duality

Maxwell Alexander Rosenlicht, The nonminimality of the differential closure .

Peter Michael Rosenthal, On an inversion theorem for the general Mehler-Fock transform pair.

Alan Saleski, Stopping times for Bernoulli automorphisms

John Herman Scheuneman, Fundamental groups of compact complete locally affine complex surfaces. II. ........................

Vashishtha Narayan Singh, Reproducing kernels and operators with a cyclic vector. I. .

Peggy Strait, On the maximum and minimum of partial sums of random variables.

J. L. Brenner, Maximal ideals in the near ring of polynomials modulo 2 .

Ernst Gabor Straus, Remark on the preceding paper: "Ideals in near rings of polynomials over a field" ..........................

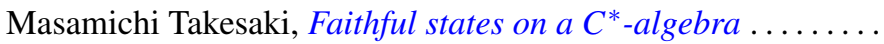

R. Michael Tanner, Some content maximizing properties of the regular simplex.

Andrew Bao-hwa Wang, An analogue of the Paley-Wiener theorem for certain

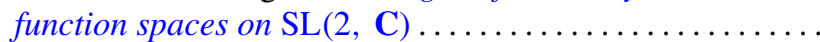

